\title{
Vibrational Sum Frequency Spectroscopic Investigation of the Azimuthal Anisotropy and Rotational Dynamics of Methyl- Terminated Silicon(111) Surfaces
}

\author{
Sergey Malyk, ${ }^{\dagger}$ Fadel Y. Shalhout, ${ }^{\dagger}$ Leslie E. O’Leary, $^{\ddagger}$ Nathan S. Lewis, ${ }^{\ddagger}$ and Alexander V. Benderskii* ${ }^{\dagger}{ }^{\dagger}$ \\ ${ }^{\dagger}$ Department of Chemistry, University of Southern California, Los Angeles, California 90089, United States \\ ${ }^{\ddagger}$ Division of Chemistry and Chemical Engineering, Beckman Institute, and Kavli Nanoscience Institute, California Institute of \\ Technology, Pasadena, California 91106, United States
}

\section{Supporting Information}

ABSTRACT: Polarization-selected vibrational sum frequency generation spectroscopy (SFG) has been used to investigate the molecular orientation of methyl groups on $\mathrm{CH}_{3}-$ terminated $\mathrm{Si}(111)$ surfaces. The symmetric and asymmetric $\mathrm{C}-\mathrm{H}$ stretch modes of the surface-bound methyl group were observed by SFG. Both methyl stretches showed a pronounced azimuthal anisotropy of the 3-fold symmetry in registry with the signal from the $\mathrm{Si}(111)$ substrate, indicating that the propeller-like rotation of the methyl groups was hindered at room temperature. The difference in the SFG line widths for the $\mathrm{CH}_{3}$ asymmetric stretch that was observed for different polarization combinations (SPS and PPP for SFG, visible, and IR) indicated that the rotation proceeded on a 1-2 ps time scale, as compared to the $\sim 100$ fs rotational dephasing of a free methyl rotor at room temperature.

\section{INTRODUCTION}

Chemical functionalization of crystalline silicon surfaces is of interest for applications in semiconductor technology, photovoltaics, ${ }^{1}$ molecular electronics, ${ }^{2,3}$ catalysis, ${ }^{4}$ and chemical and biological sensors. ${ }^{5}$ Methyl-terminated $\mathrm{Si}(111)$ surfaces, in which essentially all $\mathrm{Si}$ atop sites are terminated by methyl groups, have exhibited enhanced resistance to air oxidation relative to the $\mathrm{H}$-terminated $\mathrm{Si}(111)$ surface. ${ }^{6,7}$ Studies of methyl-terminated $\mathrm{Si}(111)$ surfaces using transmission infrared spectroscopy, ${ }^{8,9}$ low-temperature $\mathrm{STM}^{10}$ and helium atom diffraction ${ }^{11}$ have indicated that the $\mathrm{CH}_{3}$ groups are oriented perpendicular to the substrate. This conclusion is also supported by theoretical studies. ${ }^{12-14}$ Low-temperature scanning tunneling microscopic (STM) studies have shown that, at $4 \mathrm{~K}, \mathrm{CH}_{3}$ groups are immobile and are regularly oriented normal to the unreconstructed $\mathrm{Si}(111)$ surface. ${ }^{10,15}$ Although modeling indicates that the rotation of neighboring closedpacked $\mathrm{CH}_{3}$ groups might be inhibited due to steric interactions, the rotational dynamics of such systems at room temperature are not well elucidated. ${ }^{16}$

An azimuthal anisotropy of the second harmonic generation (SHG) signal has been observed for silicon surfaces with various terminations. ${ }^{17-19}$ However, the SHG signal is dominated by the above-band-gap electronic resonances in silicon, so SHG probes the symmetry of the silicon substrate (3-fold symmetry in the case of $\mathrm{Si}(111)$ ) rather than the surface-bound chemical species. Linear infrared (IR) spectroscopy has been used to characterize the vibrations of the $\mathrm{CH}_{3}$ - and $\mathrm{C}_{2} \mathrm{H}_{5}$-functionalized $\mathrm{Si}(111)$ surfaces. ${ }^{8,9}$ While the $\mathrm{C}-\mathrm{H}$ stretching and bending umbrella motions can be detected by IR absorption, the linear spectroscopy does not provide the sensitivity and information content regarding the molecular orientation and conformation that is afforded by surfaceselective nonlinear spectroscopies, such as vibrational sum frequency generation. ${ }^{20-29}$ For example, infrared spectroscopic measurements are relatively insensitive to differences between zero and small tilt angles (on the order of $10^{\circ}-20^{\circ}$ ) of the $\mathrm{CH}_{3}$ group with respect to the $\mathrm{Si}(111)$ surface normal because such changes would result in only about $10 \%$ change in the magnitude of the IR transition dipole projection onto the surface normal.

In this work, we have used vibrational sum frequency generation (SFG) spectroscopy to study the orientation of the methyl group of $\mathrm{CH}_{3}-\mathrm{Si}(111)$ surfaces. Specifically, two of the methyl vibrational modes, the symmetric $\left(r^{+}\right)$and asymmetric $\left(r^{-}\right) \mathrm{C}-\mathrm{H}$ stretches, have been investigated. SFG is a secondorder surface-selective technique in which two ultrafast laser pulses interact at the surface (or interface), to induce a secondorder polarization, $P^{(2)}(\omega)$

$$
P^{(2)}(\omega)=\chi^{(2)} E_{\text {vis }}(\omega) E_{\mathrm{IR}}(\omega)
$$

Received: July 6, 2012

Revised: November 9, 2012

Published: December 14, 2012 
where $E_{\mathrm{IR}}(\omega)$ and $E_{\mathrm{vis}}(\omega)$ are the electric fields of the infrared and visible laser pulses, respectively, and $\chi^{(2)}$ is the secondorder susceptibility. This second-order polarization acts as the source term for the radiation of the SFG signal, whose intensity is given by

$$
I^{\mathrm{SFG}}(\omega) \propto\left|P^{(2)}(\omega)\right|^{2}
$$

The second-order nonlinear susceptibility, $\chi^{(2)}$, is a property of the material and thus contains both resonant $\chi_{\mathrm{R}}^{(2)}$ (vibrational transitions) and nonresonant $\chi_{\mathrm{NR}}^{(2)}$ (off- or on-resonance electronic polarizability) contributions

$$
\chi^{(2)}=\chi_{\mathrm{R}}^{(2)}+\chi_{\mathrm{NR}}^{(2)}
$$

$\chi_{\mathrm{R}}^{(2)}$ is significantly enhanced when the IR field is resonant with a molecular vibrational mode. In contrast, $\chi_{\mathrm{NR}}^{(2)}$ does not exhibit sharp vibrational resonances and is typically small for dielectric substrates but can be large for metal surfaces.

\section{EXPERIMENTAL DETAILS}

2.1. SFG Setup. The broad-band SFG spectroscopy setup was based on a femtosecond Ti:Sapphire laser system (Spectra Physics Spitfire) that was retrofitted with a Coherent Legend regenerative amplifier cavity, pumped with a Nd:YLF laser (18 W, $1 \mathrm{kHz}$, Evolution-30, Spectra Physics), and seeded with a Ti:Sapphire oscillator $(350 \mathrm{~mW}, 82 \mathrm{MHz}$, Kapteyn-Murnane Laboratories) centered at $\sim 800 \mathrm{~nm}$ (full width at halfmaximum, fwhm $\approx 50 \mathrm{~nm}$ ). The oscillator was pumped with a Nd: $\mathrm{YVO}_{4}$ laser (2.6-3.0 W, Millenia Vs J, Spectra Physics). Sixty percent of the $4 \mathrm{~mJ}$ fundamental pulse was directed through a compressor that produced fwhm $\approx 60$ fs pulses $(1.8$ $\mathrm{mJ}, \sim 796 \mathrm{~nm}$, fwhm $\sim 27 \mathrm{~nm})$ that were used to pump an optical parametric amplifier (TOPAS-C, Light Conversion). The signal and idler pulses $(\lambda=1.1-2.6 \mu \mathrm{m})$ that were produced by the TOPAS were mixed in a difference frequency generator (NDFG, Light Conversion), to yield tunable IR pulses $\left(500-5000 \mathrm{~cm}^{-1}\right)$. The mid-IR output was $\sim 10 \mathrm{~mW}$ centered at $2900 \mathrm{~cm}^{-1}$ (C-H stretch region) with a fwhm $\sim 350 \mathrm{~cm}^{-1}$. The remaining $40 \%$ of the fundamental pulse was directed into a second compressor, to produce $\sim 60$ fs visible pulses. These pulses were then sent into a high-power airspaced etalon (TecOptics; fwhm $=17 \mathrm{~cm}^{-1}$, free spectral range $\approx 480 \mathrm{~cm}^{-1}$, finesse $\left.\approx 65\right)$ to produce a picosecond narrowbandwidth pulse that was centered near $796 \mathrm{~nm}$ (see Supporting Information, Figure S2). Two separate lenses, a $25 \mathrm{~cm}$ focal length $\mathrm{CaF}_{2}$ lens for the IR beam and a $45 \mathrm{~cm} \mathrm{BK7}$ lens for the visible beam, were used to focus the beams onto a $200 \mu \mathrm{m}$ spot on the sample surface. The IR and visible beams were incident at $66^{\circ}$ and $63^{\circ}$ from the surface normal, respectively. The laser power at the sample was $8-9 \mu \mathrm{J} /$ pulse for the IR. The intensity of the visible was adjusted by a variable density filter before the sample stage so as not to damage the sample, $<10 \mu \mathrm{J} /$ pulse for $\mathrm{CH}_{3}-\mathrm{Si}(111)$ samples. The time delay between the two pulses was varied by a joystickcontrolled translation stage (Newport VX-25, $0.1 \mu \mathrm{m}$ (0.67 fs) accuracy). The SFG signal was recollimated, spatially and frequency filtered, and focused onto the entrance slit of a 300 $\mathrm{mm}$ monochromator (Acton Spectra-Pro 300i). The signal was detected using a liquid nitrogen-cooled CCD (Princeton Instruments, Spec-10:100B, $1340 \times 100$ pixels). PPP (SFGvisible-IR), SSP, and SPS polarization combinations were used to obtain SFG spectra, as indicated below. The polarization of the visible beam was controlled by using a zero-order quartz half-wave plate $(800 \mathrm{~nm}$, CVI Melles Griot), and the IR beam polarization was controlled by using a zero-order $\mathrm{MgF}_{2}$ halfwave plate $(150-6500 \mathrm{~nm}, 5 \mathrm{~mm}$ thick, Alphalas). The SFG polarization was controlled by using a zero-order quartz halfwave plate $(670 \mathrm{~nm}$, CVI Melles Griot). To eliminate polarization contamination, polarizers were used for the IR (wire-grid holographic polarizer, extinction ratio >300:1) and SFG beams (polarizing beamsplitter cube, extinction ratio $>500: 1)$.

The spectrum of the narrow-band visible pulse was recorded, using the same signal collection optics and the same monochromator, by replacement of the sample surface with a reflective gold substrate (BioGold Microarray Slides, Thermo Scientific). The spectrum of the narrow-bandwidth visible pulse was recorded using the same grating and CCD as for SFG detection. The spectra of the IR pulses were measured using an IR grating blazed at $5 \mu \mathrm{m}$ and a liquid nitrogen-cooled $\mathrm{HgCdTe}$ detector (IR Associates). The temporal profiles of the compressed fundamental $800 \mathrm{~nm}$ pulses were determined, using a home-built single-shot autocorrelator, to have fwhm 60 fs. The compressed fundamental pulses were then used to characterize the time width and chirp of the broadband (BB) IR pulses using the SFG cross-correlation on a nonresonant substrate $(\mathrm{Au})$. The temporal profiles of the narrow-band picosecond visible pulses that were produced by the etalon were measured by scanning the femtosecond IR pulse across the visible pulse and recording the SFG cross-correlation signal from a nonresonant Au substrate.

2.2. Sample Preparation and Handling. $\mathrm{CH}_{3}$-terminated $\mathrm{Si}(111)$ surfaces were prepared and characterized as described previously. ${ }^{8,30-32}$ The samples were rinsed with water, acetone, methanol, and again with water. The samples were then dried with inert gas and heated at $450{ }^{\circ} \mathrm{C}$ for 3-5 days under vacuum, to remove adventitious carbon (organic impurities) prior to SFG studies. To confirm the removal of the impurities, a SFG spectrum was taken before and after the cleaning procedure. Upon cleaning, the methylene $\left(-\mathrm{CH}_{2}-\right)$ vibrational peaks from impurities could not be detected, and only the $\mathrm{CH}_{3}$ symmetric and asymmetric peaks remained. Additional heating of the sample did not alter the sample, as evidenced by a lack of change in the $\mathrm{CH}_{3}$ peaks. A SFG spectrum in the $\mathrm{Si}-\mathrm{H}$ stretching spectral region $\left(\sim 2100 \mathrm{~cm}^{-1}\right)$ confirmed the absence of the H-terminated Si sites and was indicative of $100 \%$ methyl monolayer coverage of the Si surface.

All SFG measurements were performed in dry air obtained from the FTIR purge gas generator. Data acquisition was completed within $3 \mathrm{~h}$. The SFG spectra were reproducible for different spots on the sample, indicating that there was no damage due to the laser beams, and for different samples. The SFG spectra shown below represent one of the samples, and the azimuthal dependences combine data collected for three samples. Samples that were exposed to ambient air for longer than a few hours became contaminated with hydrocarbons, evidenced by the appearance of $-\mathrm{CH}_{2}-$ vibrational peaks in SFG spectra, indicating that the methyl-terminated Si surface is strongly lipophilic.

2.3. Orientational Anisotropy and Rotational Dynamics. The rotational anisotropy of the samples was determined for PPP and SSP polarization combinations for $0 \mathrm{fs}$ IR-visible delay ( 2 and 5 min exposure times, respectively). PPP spectra for $300 \mathrm{fs}$ delay were recorded with an exposure time of $5 \mathrm{~min}$. A graduated rotation stage (estimated accuracy $\pm 0.5^{\circ}$ ) was used for azimuthal rotation of the sample (in the plane of the 
sample surface). The rotational stage was aligned such that the rotation axis coincided with the spot where the IR and visible beams overlapped on the sample surface. This alignment procedure ensured that rotation of the stage would not affect the position of the irradiated region of the sample.

To study the in-plane rotational dynamics of the methyl groups, several SFG spectra were collected and averaged for PPP (5 min exposure times) and SPS (30 or 40 min exposure times) polarization combinations at a $300 \mathrm{fs}$ IR-vis delay.

2.4. Signal Processing. The CCD image was processed using WinSpec (Roper Scientific). The SFG signal for all experiments was focused onto four or five pixel strips in the vertical $(\mathrm{Y})$ direction. A CCD pixel binning of $3 \mathrm{X} \times 4 \mathrm{Y}$ or $3 \mathrm{X} \times$ $5 \mathrm{Y}$ was used in all experiments. A background correction was performed by subtracting a nonilluminated region of the CCD (e.g., strip 16) from the SFG signal strip (e.g., strip 15). Spikes due to cosmic X-rays were removed using an internal discriminator option within the WinSpec program. The IR frequencies were calculated by subtracting the central frequency of the narrow-band visible pulse from the SFG frequency.

\section{RESULTS}

SFG spectra of the $\mathrm{CH}_{3}-\mathrm{Si}(111)$ surface were recorded at room temperature, for PPP (SFG-vis-IR) and SSP polarization combinations, for a full $360^{\circ}$ rotation in the plane of the sample. The spectra covered the $\mathrm{C}-\mathrm{H}$ vibrational stretch region from 2800 to $3000 \mathrm{~cm}^{-1}$. Two peaks, corresponding to the symmetric and asymmetric stretch, respectively, of the methyl group were observed. All of the SFG spectra were fitted to the relationship ${ }^{33}$

$$
\begin{aligned}
I^{\mathrm{SFG}}(\omega)= & \left|\chi_{\mathrm{eff}}^{(2)}(\omega) E_{\mathrm{IR}}(\omega) \circ E_{\mathrm{vis}}(\omega)\right|^{2} \\
& \left.=\mid \chi_{\mathrm{NR}}^{(2)}+\chi_{\mathrm{R}}^{(2)}\right)\left.E_{\mathrm{IR}}(\omega) \circ E_{\mathrm{vis}}(\omega)\right|^{2} \\
& \approx\left|A_{\mathrm{NR}} e^{i \phi}+\sum_{j=1}^{N} \frac{B_{j}}{\omega-\omega_{j}+i \Gamma_{j}}\right|^{2} e^{-\left(\omega-\omega_{\mathrm{g}}\right)^{2} / \sigma^{2}}
\end{aligned}
$$

which assumes a Gaussian spectral profile for the broad-band mid-infrared pulse with central frequency $\omega_{\mathrm{g}}$ and width $\sigma_{\mathrm{g}}$, and incorporates a convolution (o) with the narrow-band visible pulse into the Lorentzian line shapes of the resonances. The vibrationally nonresonant term, $\chi_{\mathrm{NR}}^{(2)}=A_{\mathrm{NR}} e^{i \phi}$, with an amplitude $A_{\mathrm{NR}}$ and a phase $\phi$ relative to the resonant contribution, represents the electronically resonant response of the silicon substrate because both the visible and the VSFG photon energies are above the Si band gap. Equation 4 assumes that this contribution to $\chi_{\mathrm{NR}}^{(2)}$ is spectrally flat within the relatively narrow $\left(\sim 200 \mathrm{~cm}^{-1}\right)$ frequency range corresponding to SFG wavelengths between 645 and $654 \mathrm{~nm}$. The resonant term $\chi_{\mathrm{R}}^{(2)}=\sum_{j=1}^{N}\left[B_{j} /\left(\omega-\omega_{j}+i \Gamma_{j}\right)\right]$ describes Bloch-type (exponential) dephasing for each vibrational mode $j$, by a Lorentzian line shape with a line width $\Gamma_{j}$, an amplitude $B_{j} / \Gamma_{j}$, and a central frequency $\omega_{j}$.

A time-delay technique introduced by Lagutchev et al., ${ }^{34}$ in which the asymmetry of the visible pulse in the time domain (see Supporting Information, Figure S2) is used to up-convert mainly the slower resonant contribution when the IR-visible time delay is judiciously chosen, was used to decouple the nonresonant and resonant signals. As shown in Figure S3 in the Supporting Information, this technique is particularly advantageous when a considerable nonresonant SFG signal is present, as in the PPP spectra. However, a time delay was not used for the SSP spectra because the signal was weaker at delayed times, and longer exposure times were thus required to obtain acceptable signal-to-noise.

Figure 1 shows a series of PPP spectra at the $0 \mathrm{fs}$ IR-visible time delay, for azimuthal angles $\varphi$ varying from $0^{\circ}$ to $120^{\circ}$. This

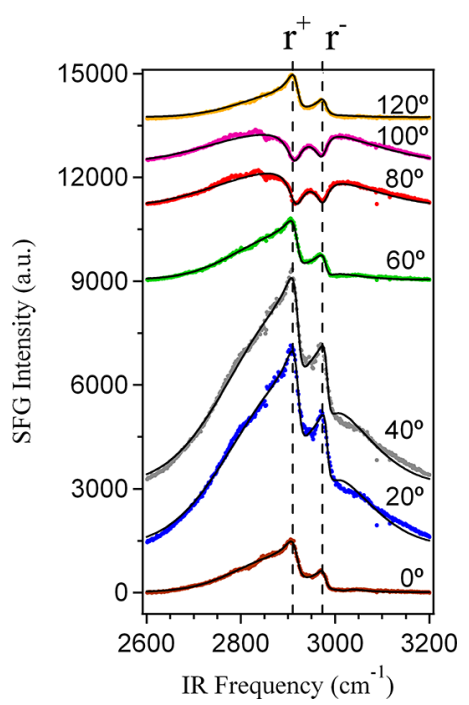

Figure 1. SFG PPP polarized spectra ( 0 fs delay) for rotation angles of $0^{\circ}$ (brown dots), $20^{\circ}$ (blue), $40^{\circ}$ (gray), $60^{\circ}$ (green), $80^{\circ}$ (red), $100^{\circ}$ (purple), and $120^{\circ}$ (orange). Only one rotational period is shown for clarity. Solid black lines are fits.

pattern repeated itself for azimuthal angles from $120^{\circ}$ to $240^{\circ}$ and again from $240^{\circ}$ to $360^{\circ}$, indicating a 3-fold in-plane symmetry. The PPP spectra showed the $\mathrm{CH}_{3}$ symmetric stretch $\left(r^{+}\right)$, at $2912 \mathrm{~cm}^{-1}$, and the $\mathrm{CH}_{3}$ asymmetric stretch $\left(r^{-}\right)$, at $2976 \mathrm{~cm}^{-1}$. The solid black lines in Figure 1 are the fits using eq 4, with the fitting parameters for each azimuthal angle listed in Table 1 of the Supporting Information.

Figure 2 shows the azimuthal rotational anisotropy of the nonresonant amplitude $A_{\mathrm{NR}}$ (red squares) for the full range of angles, $\varphi$, from $0^{\circ}$ to $360^{\circ}$, that were obtained from the fits using eq 4. Also shown is the fit (blue line) that describes the rotational anisotropy

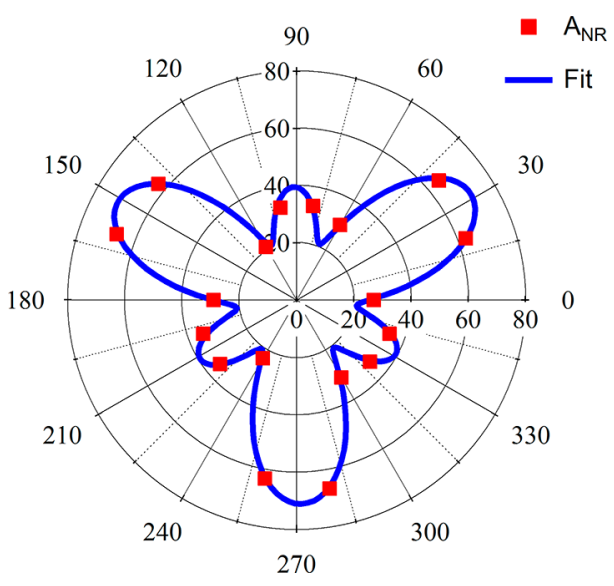

Figure 2. Polar plot showing the azimuthal dependence of the nonresonant amplitude, $A_{\mathrm{NR}}$, on the in-plane rotation angle, $\varphi$, changing counter-clockwise from $0^{\circ}$ to $360^{\circ}$, for PPP spectra (0 fs delay). 


$$
A(\varphi)=\sqrt{\left(a+c \cos \left(3 \varphi+\varphi_{0}\right)\right)^{2}+k^{2}}
$$

where $a$ and $c$ are the isotropic and anisotropic contributions to the response, respectively; $k$ is a term to account for incoherent background (e.g., scattering); and $\varphi$ is the azimuthal angle, with a phase correction of $\varphi_{0}$.

Because of the strong nonresonant signal at 0 fs delay observed in the PPP spectra, the resonant amplitudes for the methyl stretches could not be determined accurately. A $300 \mathrm{fs}$ delay between the IR and visible pulses was used to suppress the nonresonant background signal $\chi_{\mathrm{NR}}^{(2)}$. Figure 3 shows the

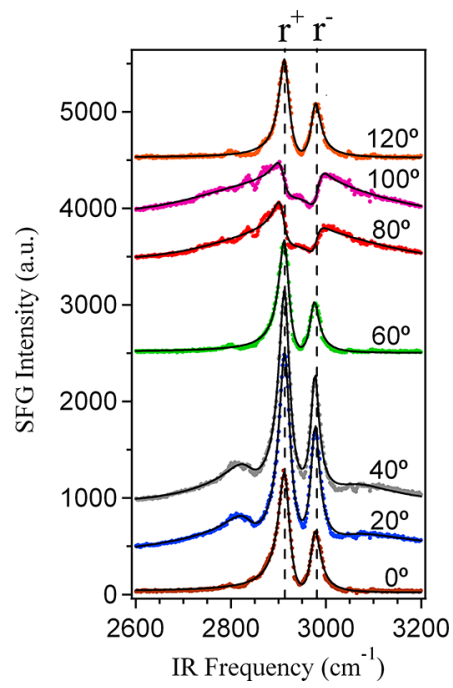

Figure 3. SFG PPP polarized spectra (300 fs delay) for rotation angles $0^{\circ}$ (brown dots), $20^{\circ}$ (blue), $40^{\circ}$ (gray), $60^{\circ}$ (green), $80^{\circ}$ (red), $100^{\circ}$ (purple), and $120^{\circ}$ (orange). Only one rotational period is shown for clarity. The solid black lines are fits.

PPP spectra, at 300 fs delay, for the same range of azimuthal angles as in Figure $1,0^{\circ}<\varphi<120^{\circ}$ (again, the pattern repeated itself twice more, for $120^{\circ}<\varphi<240^{\circ}$ and $240^{\circ}<\varphi<360^{\circ}$ ). The fitting parameters for each azimuthal angle are listed in Table 2 in the Supporting Information. Figures 4 and 5 present the resonant amplitudes for the symmetric and asymmetric $\mathrm{CH}_{3}$ stretch, $B\left(r^{+}\right) / \Gamma\left(r^{+}\right)$and $B\left(r^{-}\right) / \Gamma\left(r^{-}\right)$(red squares),

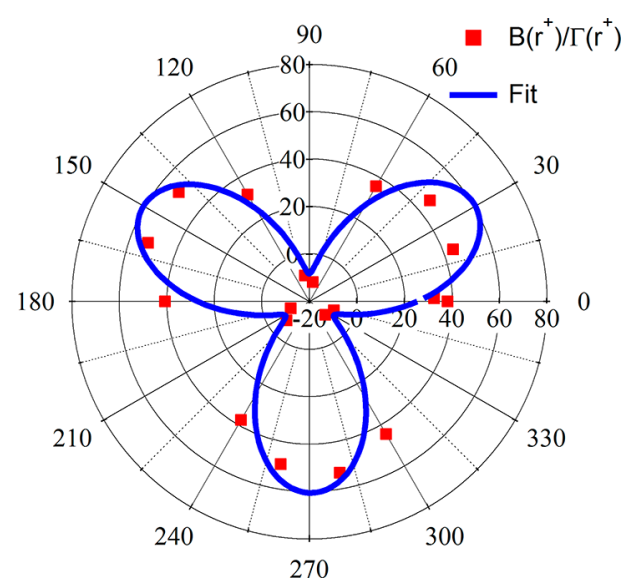

Figure 4. Polar plot showing the azimuthal dependence of the $\mathrm{CH}_{3}$ symmetric stretch amplitude $B\left(r^{+}\right)$of the PPP spectra (300 fs delay) normalized by the $\Gamma\left(r^{+}\right)$on the in-plane rotation angle $\varphi$ changing counter-clockwise from $0^{\circ}$ to $360^{\circ}$.

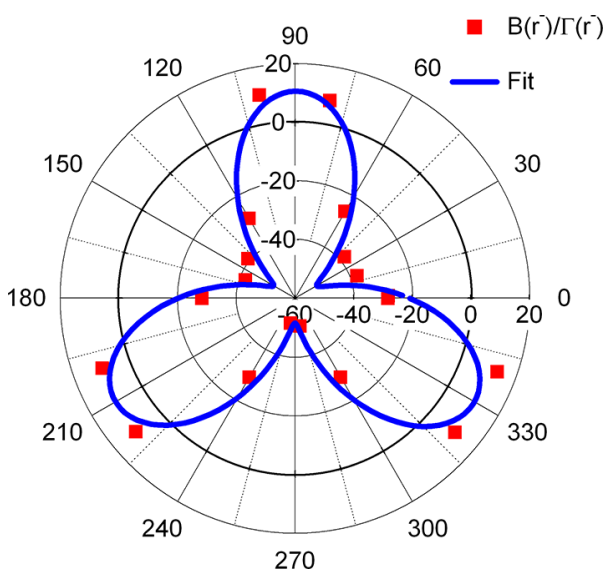

Figure 5. Polar plot showing the azimuthal dependence of the $\mathrm{CH}_{3}$ asymmetric stretch amplitude $B\left(r^{-}\right)$normalized by $\Gamma\left(r^{-}\right)$on the inplane rotation angle $\varphi$, changing counter-clockwise from $0^{\circ}$ to $360^{\circ}$, for PPP spectra (300 fs delay).

obtained from the fits (eq 4) for each azimuthal angle. The model (shown as blue lines in Figures 4 and 5) that was used to describe the resonant amplitude anisotropy is

$$
B(\varphi)=a+c \cos \left(3 \varphi+\varphi_{0}\right)
$$

where the definitions of the terms are the same as in eq 5 .

Figure 6 shows a series of SSP spectra, at 0 fs delay, for one rotational period. Only the $\mathrm{CH}_{3}$ symmetric stretch $\left(r^{+}\right)$at

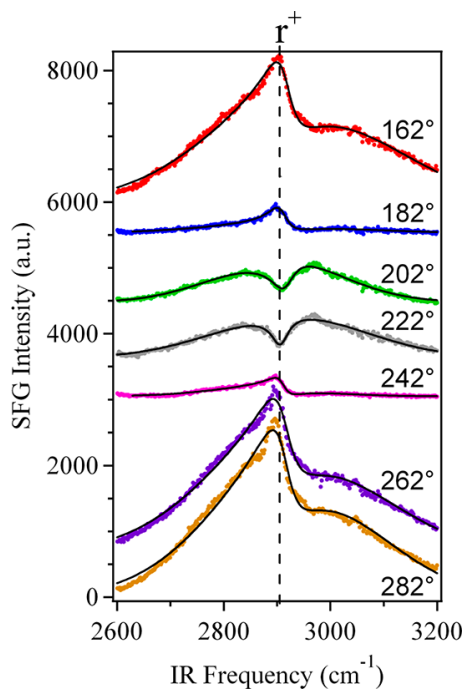

Figure 6. SFG SSP polarized spectra (0 fs delay) for rotation angles $162^{\circ}$ (red dots), $182^{\circ}$ (blue dots), $202^{\circ}$ (green), $222^{\circ}$ (gray), $242^{\circ}$ (pink), $262^{\circ}$ (purple), and $282^{\circ}$ (brown). Only one rotational period is shown for clarity. Solid black lines are fits.

$\sim 2912 \mathrm{~cm}^{-1}$ was observed in the SSP spectra. The nonresonant, $A_{\mathrm{NR}}$, and resonant, $B\left(r^{+}\right) / \Gamma\left(r^{+}\right)$, amplitudes were obtained from the fits to the spectra using eq 4 and are plotted in Figures 7 and 8, respectively (the complete list of SSP spectral fitting parameters is given in the Supporting Information, Table 3). Equations 5 and 6 were used to describe the nonresonant and resonant anisotropy in the SSP spectra. A list of the fitting parameters used in eqs 5 and 6 for both PPP and SSP spectra is provided in the Supporting Information, Table 4. 


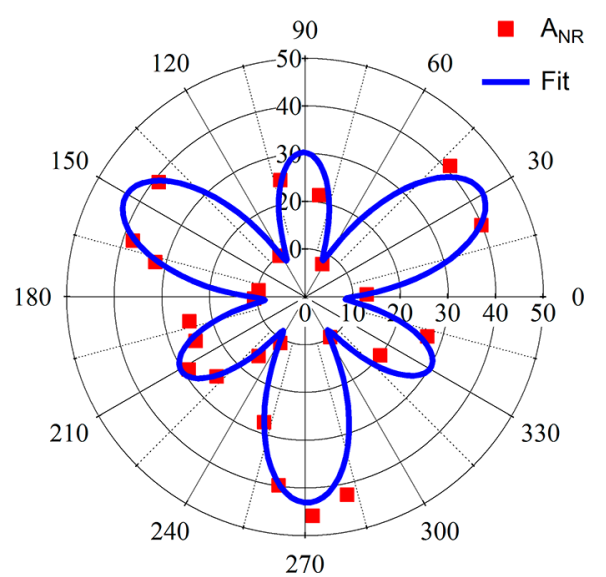

Figure 7. Polar plot showing the azimuthal dependence of the nonresonant amplitude $A_{\mathrm{NR}}$ on the in-plane rotation angle $\varphi$ changing counter-clockwise from $0^{\circ}$ to $360^{\circ}$, for SSP spectra.

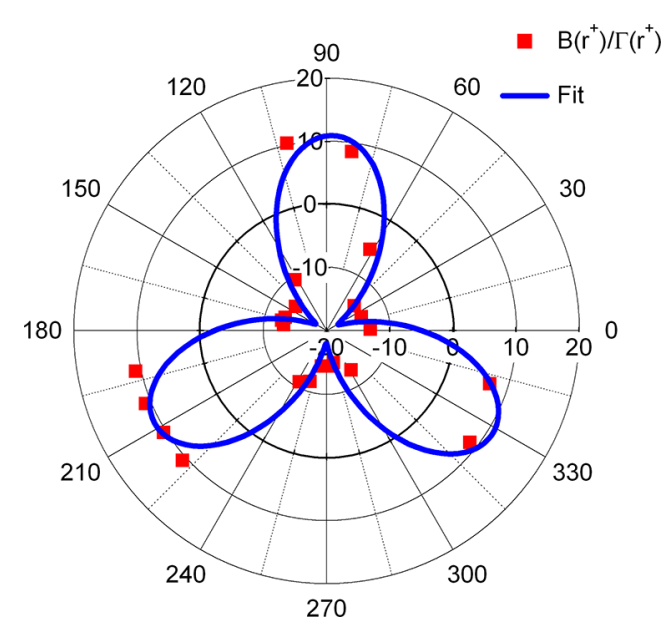

Figure 8. Polar plot showing the azimuthal dependence of the $\mathrm{CH}_{3}$ symmetric stretch amplitude $B\left(r^{+}\right)$normalized by $\Gamma\left(r^{+}\right)$on the inplane rotation angle $\varphi$ changing counter-clockwise from $0^{\circ}$ to $360^{\circ}$, for SSP spectra.

Figure 9 shows the SFG spectra for the PPP (red dots) and SPS (blue dots) polarization combinations, averaged over four sets of PPP and SPS spectra recorded with a 300 fs delay. A broadening of the spectral line shape for the $\mathrm{CH}_{3}$ asymmetric

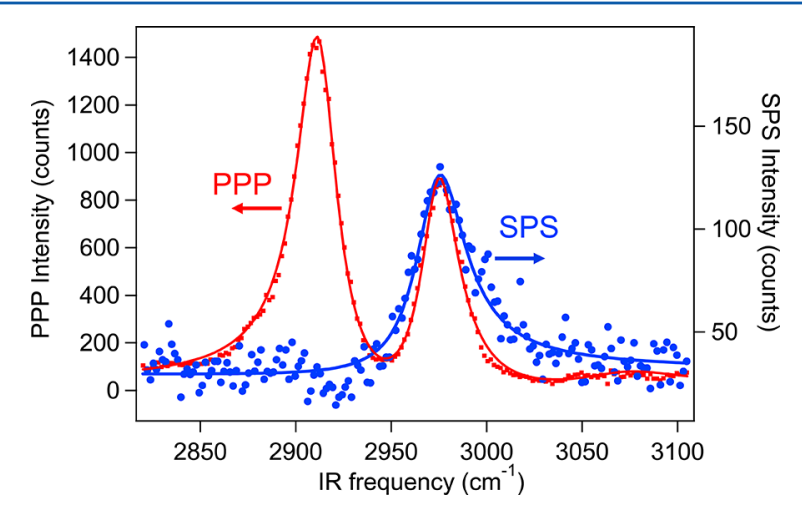

Figure 9. Broadening of the line width $\Gamma$ for the $\mathrm{CH}_{3}$ asymmetric stretch $\left(r^{-}\right)$at $2976 \mathrm{~cm}^{-1}$ for the SPS spectrum (blue) compared to the PPP (red) polarized SFG spectrum. Solid blue and red lines are fits of the SPS and PPP spectra, respectively, to eq 4. stretch $\left(r^{-}\right)$vibrational mode was observed for the SPS spectrum relative to the PPP spectrum. The spectral fits that were performed using eq 4 are shown as solid lines. These measurements were performed at a $120^{\circ}$ rotational angle, for which the nonresonant background was at its minimum. The asymmetric stretch line width for the PPP spectra was $\Gamma_{\mathrm{PPP}}\left(r^{-}\right)$ $=12.4 \pm 0.5 \mathrm{~cm}^{-1}$ and for the SPS spectra was $\Gamma_{\mathrm{SPS}}\left(r^{-}\right)=15.9$ $\pm 1.0 \mathrm{~cm}^{-1}$. The difference in the line widths, $3.5 \pm 1.5 \mathrm{~cm}^{-1}$, was interpreted as rotational dephasing, as discussed in detail below.

\section{DISCUSSION}

4.1. Vibrationally Nonresonant Response of the Silicon. A phenomenological macroscopic theory of the second harmonic generation (SHG) electronic response of a surface of a cubic centrosymmetric crystal was developed by Sipe et al. $^{18}$ This theory does not make any assumptions regarding the microscopic physical properties of the surface or bulk response tensor. The theory is rigorous in the sense that it is purely based on the macroscopic symmetry of the surface and of the bulk of the crystal. ${ }^{16}$ The approach provides a convenient framework with which to analyze the SFG signals from the $\mathrm{CH}_{3}-\mathrm{Si}(111)$ surface. The key concepts and equations, specifically adapted for understanding the vibrational sum frequency response of the $\mathrm{CH}_{3}-\mathrm{Si}(111)$ surface, are briefly summarized below.

The effective second-order polarization $P^{(2)}(\omega)$ contains contributions from the surface and from the bulk of Si crystal. Each of these contributions has isotropic and anisotropic components, based on the crystal symmetry. The bulk contribution to the effective polarization, in the case of a homogeneous medium excited by a single transverse plane wave, consists of nonlocal electric quadrupole as well as magnetic dipole terms (the lowest-order surviving multipole terms) and can be written as ${ }^{17,18,35,36}$

$$
P_{i}^{(2)}=\gamma \nabla_{i}\left(E^{\mathrm{IR}} \cdot E^{\mathrm{vis}}\right)+\zeta_{i i i i} E_{i}^{\mathrm{IR}} \nabla_{i} E_{i}^{\mathrm{vis}}
$$

where $\gamma$ and $\zeta$ are phenomenological constants. The first term is isotropic with respect to the crystal orientation, while the second term has both isotropic and anisotropic components (here we neglect the term that is nonlocal in the IR field). For a $\mathrm{Si}(111)$ surface that has 3 -fold rotation symmetry, the SFG fields that are generated outside of the medium have a form ${ }^{17,18}$

$$
E^{\mathrm{SFG}}=(a+c \cos 3 \varphi) E^{\mathrm{vis}} E^{\mathrm{IR}}
$$

where $\varphi$ is the azimuthal angle within the (111) plane, and $a$ and $c$ represent the isotropic and anisotropic response, respectively.

We only consider the SSP and PPP polarization combinations that are relevant to our experimental conditions. The SFG fields that are generated by the bulk response for these two polarization combinations are

$$
\begin{aligned}
& E_{p}^{\mathrm{SFG}}=A_{p}\left(\zeta D\left(a_{\text {bulk }}^{p}+c_{\text {bulk }}^{p} \cos 3 \varphi\right)+\gamma F_{\mathrm{S}}\right) E_{p}^{\mathrm{vis}} E_{p}^{\mathrm{IR}} \\
& E_{s}^{\mathrm{SFG}}=A_{s} \zeta D\left(a_{\text {bulk }}^{s}+c_{\text {bulk }}^{s} \cos 3 \varphi\right) E_{s}^{\mathrm{vis}} E_{p}^{\mathrm{IR}}
\end{aligned}
$$

where $a_{\text {bulk }}^{s, p}$ and $c_{\text {bulk }}^{s, p}$ are bulk isotropic and anisotropic coefficients, respectively. $F_{S}$ is the sine of the angle of the SFG beam in the silicon, and $A_{p}, A_{s}$, and $D$ are constants that contain the angle of incidence and the optical properties (refractive indices) of the interface. ${ }^{18}$ Calculation of the isotropic and anisotropic bulk coefficients, ${ }^{18} a_{\text {bulk }}^{s, p}$ and $c_{\text {bulk }}^{s, p}$ 
involves only the incident angles and the dielectric constant of the medium at IR, visible, and SFG frequencies (details are presented in the Supporting Information). Essentially, two adjustable parameters, $\gamma$ and $\zeta$, describe the nonlinear response of the bulk medium and thus can change the bulk contribution to the signal.

The surface contribution to the SFG signal is primarily dipolar because the inversion symmetry is broken on $\mathrm{CH}_{3}-$ Si(111) surfaces. ${ }^{17,18}$ The symmetry of the SFG nonlinear susceptibility tensor is assumed to be determined by the symmetry of the $\mathrm{Si}(111)$ surface. If only the first layer of $\mathrm{Si}$ atoms is taken into account, the symmetry is $C_{6 v}{ }^{17,18}$ however, the symmetry lowers to $C_{3 v}$ if additional surface layers are considered. ${ }^{17,18}$ The nonlinear surface susceptibility tensor for the $\mathrm{Si}(111)$ surface should satisfy $C_{3 v}$ symmetry and thus has four independent elements, comprised of three isotropic terms and one anisotropic term. ${ }^{17}$ The second-order polarization for the $\operatorname{Si}(111)$ surface, when the $z$-axis is perpendicular to the surface and the $y$-axis is perpendicular to the plane of symmetry, can be written as ${ }^{17,18}$

$$
\left|\begin{array}{l}
P_{x}^{(2)} \\
P_{y}^{(2)} \\
P_{z}^{(2)}
\end{array}\right|=\left|\begin{array}{cccccc}
\partial_{11} & -\partial_{11} & 0 & 0 & \partial_{15} & 0 \\
0 & 0 & 0 & \partial_{15} & 0 & -\partial_{11} / 2 \\
\partial_{31} & \partial_{31} & \partial_{33} & 0 & 0 & 0
\end{array}\right|\left|\begin{array}{c}
E_{x}^{\mathrm{vis}} E_{x}^{\mathrm{IR}} \\
E_{y}^{\mathrm{vis}} E_{y}^{\mathrm{IR}} \\
E_{z}^{\mathrm{vis}} E_{z}^{\mathrm{IR}} \\
E_{y}^{\mathrm{vis}} E_{z}^{\mathrm{IR}} \\
E_{x}^{\mathrm{vis}} E_{z}^{\mathrm{IR}} \\
E_{x}^{\mathrm{vis}} E_{y}^{\mathrm{IR}}
\end{array}\right|
$$

where $\partial_{15}, \partial_{31}$, and $\partial_{33}$ are isotropic terms and $\partial_{11}$ is an anisotropic term. As can be seen from eq 10, these constants are simply related to the elements of the second-order SFG susceptibility tensor $\chi^{(2)}$ by

$$
\chi_{z z z}^{(2)}=\partial_{33}, \chi_{z x x}^{(2)}=\partial_{31}, \chi_{x x z}^{(2)}=\partial_{15}, \chi_{x x x}^{(2)}=\partial_{11}
$$

The SFG surface responses for PPP and SSP polarizations have the form ${ }^{18}$

$$
\begin{aligned}
& E_{p}^{\mathrm{SFG}}=A_{p}\left(a_{\text {surf }}^{p}+c_{\text {surf }}^{p} \cos 3 \varphi\right) E_{p}^{\mathrm{vis}} E_{p}^{\mathrm{IR}} \\
& E_{s}^{\mathrm{SFG}}=A_{s}\left(a_{\text {surf }}^{s}+c_{\text {surf }}^{s} \cos 3 \varphi\right) E_{s}^{\mathrm{vis}} E_{p}^{\mathrm{IR}}
\end{aligned}
$$

where, as outlined in the Supporting Information, the $a_{\text {surf }}^{s, p}$ and $c_{\text {surf }}^{s, p}$ are surface isotropic and anisotropic coefficients that contain the $\partial$ coefficients defined in eq 10, the incidence beam angles, and the dielectric constants. ${ }^{18}$ The constants $A_{p}$ and $A_{s}$ are the same as in eq 9 .

Combining the bulk, eq 9, and surface, eq 12, expressions, the isotropic and anisotropic contributions to the total SFG signal for PPP and SSP polarization combinations are thus

$$
\begin{aligned}
& E_{p}^{\mathrm{SFG}}=A_{p}{ }^{\prime}\left(1+R_{\mathrm{PPP}} \cos 3 \varphi\right) E_{p}^{\mathrm{vis}} E_{p}^{\mathrm{IR}} \\
& E_{s}^{\mathrm{SFG}}=A_{s}{ }^{\prime}\left(1+R_{\mathrm{SSP}} \cos 3 \varphi\right) E_{s}^{\mathrm{vis}} E_{p}^{\mathrm{IR}}
\end{aligned}
$$

where we have introduced the ratio $R_{\mathrm{PPP}, \mathrm{SSP}}$ of the anisotropic to isotropic components that determines the modulation depth in the experimentally measured azimuthal angular dependence of the SFG signal

$$
\begin{aligned}
& R_{\mathrm{PPP}}=\left(\zeta D c_{\text {bulk }}^{p}+c_{\text {surf }}^{p}\right) /\left(\zeta D a_{\text {bulk }}^{p}+\gamma F_{s}+a_{\text {surf }}^{p}\right) \\
& R_{\text {SSP }}=\left(\zeta D c_{\text {bulk }}^{s}+c_{\text {surf }}^{s}\right) /\left(\zeta D a_{\text {bulk }}^{s}+a_{\text {surf }}^{s}\right)
\end{aligned}
$$

To compare the amplitudes of the PPP and of the SSP signals, we also define the ratio of the anisotropic parts for the SSP and PPP polarizations

$$
c_{\mathrm{PPP}} / c_{\mathrm{SSP}}=\left(\zeta D c_{\text {bulk }}^{p}+c_{\text {surf }}^{p}\right) /\left(\zeta D c_{\text {bulk }}^{s}+c_{\text {surf }}^{s}\right)
$$

The ratio of the isotropic parts of the PPP and SSP signals can be obtained from eqs 15 and 14 .

Figures 2 and 7 show the azimuthal dependence of the nonresonant amplitude of the $\mathrm{CH}_{3}-\mathrm{Si}(111) \mathrm{SFG}$ signal for the PPP and SSP polarization combinations. The SFG amplitudes clearly showed a 3-fold dependence on the azimuthal angle, with the same phase in both cases. The nonresonant SFG signal should be dominated by the response of the silicon itself (not the surface methyls) and thus is expected to be well described by the phenomenological model outlined above. Several groups have measured the azimuthal dependence of the second harmonic generation (SHG) for the $\mathrm{Si}(111)$ surface. ${ }^{17-19}$ In particular, Mitchell et al. ${ }^{19}$ studied the behavior of the isotropic $a$ and anisotropic $c$ parameters as a function of the surface functionalization and of the probe wavelength. $\mathrm{H}-\mathrm{Si}(111)$ surfaces exhibit isotropic and anisotropic parameters with a ratio $R_{\mathrm{PPP}}$ changing from $\sim 4.6$ for $830 \mathrm{~nm}$ fundamental excitation to $\sim 1.3$ for $775 \mathrm{~nm}$ excitation. For $\mathrm{CH}_{3}-\mathrm{Si}(111)$, an $R_{\text {PPP }}$ value of $\sim 2.9$ was obtained from the fit of the PPP nonresonant SFG amplitude for the $796 \mathrm{~nm}$ visible and the 650 $\mathrm{nm}$ SFG wavelength, which is well in the range of values reported by Mitchell et al. ${ }^{19}$ The nonresonant amplitude for the SSP SFG signal (see Figure 7) had $R_{\mathrm{SSP}} \sim 5.1$ and had the same phase $\varphi_{0}$ as the PPP SFG anisotropic part. Additionally, the ratio of anisotropic parts $c_{\mathrm{PPP}} / c_{\mathrm{SSP}}$ was observed to be $\sim 2.3$. The parameters for the $\mathrm{Si}(111)$ surface and bulk nonlinear response can be obtained from eqs 13 and 14, with a total of six independent parameters in the model: four for the surface response, $\partial_{15}, \partial_{31}, \partial_{33}$, and $\partial_{11}$, and two for the bulk response, $\gamma$ and $\zeta$. However, only three independent parameters that characterize the anisotropy can be obtained experimentally, eqs 14 and 15. Thus, all of the model parameters can not be determined, but the experiment can constrain some of their ratios. One possible set of the model values is listed in Table 5 of the Supporting Information. Overall, the obtained values are close to the ones reported by Mitchell et al. ${ }^{19}$

4.2. Resonant Response of the Surface-Bound Methyl Groups. The vibrationally resonant SFG signals contain information on the molecular orientation and on the dynamics 
of the $\mathrm{CH}_{3}$ groups at the $\mathrm{Si}(111)$ surface. Even without a detailed analysis, the pronounced 3-fold azimuthal anisotropy of the $\mathrm{CH}_{3}$ resonant signal (Figures 4, 5, and 8) observed in both the PPP and SSP spectra suggests that the methyl groups are well oriented and preserve the azimuthal symmetry of the $\mathrm{Si}(111)$ surface; i.e., they do not freely rotate at room temperature.

The "standard" SFG orientational analysis assumes a threelayer model for the interface ${ }^{22,37}$ and calculates the resonant contribution to $\chi^{(2)}$ by assuming a molecular hyperpolarizability tensor $\beta^{(2)}$ for a given vibrational mode. The tensor $\beta^{(2)}$ is then converted from the molecular frame $(a, b, c)$ into the lab frame $(x, y, z)$, where the $z$-axis is usually chosen to be normal to the sample surface. ${ }^{22,38,39}$ This approach has been applied to access the orientational analysis of the SFG spectra, in particular to azimuthally anisotropic systems such as rubbed polymer surfaces. ${ }^{40-42}$ The hyperpolarizability tensors for the symmetric and asymmetric stretch modes of the methyl group have been estimated from experimental SFG measurements on molecules with long alkane chains ${ }^{21,27,41,43-46}$ and can be approximated by a bond-additivity model. ${ }^{43,47}$ The SFG hyperpolarizabilities of the $\mathrm{C}-\mathrm{H}$ stretches of $\mathrm{CH}_{3} \mathrm{OH}$ have been rigorously modeled computationally. ${ }^{48,49}$ Because the methyl group $\mathrm{C}-\mathrm{H}$ stretches are relatively weakly coupled to other $\mathrm{C}-\mathrm{H}$ stretching modes, the hyperpolarizability tensors $\beta^{(2)}$ are generally assumed to be similar for terminal methyl groups on long-chain alcohols, carboxylic acids, and alkane thiols.

However, use of the literature values for $\beta^{(2)}$ of the terminal methyl group did not allow reproduction of the observed azimuthal dependence of the resonant SFG signal on the $\mathrm{CH}_{3}-$ $\mathrm{Si}(111)$ surface. Notably, the molecular hyperpolarizability tensor is a product of the vibrational transition dipole moment and the Raman polarizability tensor

$$
\beta_{l m n}^{i} \propto \frac{\partial \alpha_{l m}}{\partial q_{i}} \frac{\partial \mu_{n}}{\partial q_{i}}
$$

where $q_{i}$ is the normal coordinate of the $i$-th vibrational mode and the indices $l, m$, and $n$ represent the axes of the molecular frame $(a, b, c)$, with the $c$ axis usually chosen to be the main symmetry axis. Because of the $C 3 v$ symmetry of the methyl group, the IR transition dipole for the symmetric stretch (ss) is along the $C_{3}$ axis $(c)$, and the Raman polarizability tensor is isotropic about the symmetry axis, such that $\beta_{a a c}^{\text {ss }}=\beta_{b b c}^{\text {ss }}{ }^{37}$ Hence, if the $C_{3}$ axis of the methyl group is normal to the surface, no azimuthal anisotropy of the symmetric stretch signal can exist, regardless of the in-plane orientation of the methyl group (e.g., either isotropic random azimuthal orientation or locked orientation in registry with the silicon substrate). Thus, only asymmetric stretch (as) anisotropy can be present, for all anisotropic (e.g., 3-fold) azimuthal distributions of the methyl group. This expectation is clearly contradicted by the experimental observations.

We also considered a distribution of the methyl orientations along the Euler angles $(\theta, \psi, \varphi)$ that define the orientation of the molecular frame $(a, b, c)$ relative to the lab frame $(x, y, z)$. The distribution along the azimuthal angle $\varphi$ was taken to be a sum of three delta functions shifted by $120^{\circ}$ relative to each other, and no torsional motion was allowed (delta-function distribution along the torsion angle $\psi$ ). Several distributions of the tilt angle $\theta$, including a delta-function centered at $\theta_{0}$, a Gaussian centered at $\theta_{0}$, and a bimodal distribution with two subpopulations centered at 0 and $\theta_{0}$, were tested. The macroscopic susceptibility $\chi^{(2)}$ was then calculated by rotating $\beta^{(2)}$ from the molecular frame to the lab frame and averaging over the orientational distribution ${ }^{22,38,39,43}$

$$
\chi_{i j k}^{(2)}=N_{S} \sum_{l, m, n}\left\langle R_{i j k, l m n}(\theta, \psi, \varphi)\right\rangle_{\theta, \varphi, \psi} \beta_{l m n}^{(2)}
$$

In all cases, as expected, no azimuthal anisotropy of the symmetric stretch was calculated for $\mathrm{CH}_{3}$ groups oriented normal to the surface $\left(\theta_{0}=0\right)$. A significant tilt $\left(\theta_{0}>50^{\circ}\right)$ of the $\mathrm{CH}_{3}$ groups was required to obtain any substantial amplitude of azimuthal anisotropy for the simulated $\mathrm{CH}_{3}$ symmetric stretch $\left(r^{+}\right)$signal. Furthermore, the experimental modulation depth in the azimuthal dependence of the $r^{+}$signal in the PPP and SSP spectra (Figures 4 and 8) could not be reproduced even for $\theta_{0}=50^{\circ}$, a situation for which a substantial fraction of $\mathrm{CH}_{3}$ groups would be lying flat on the surface. The 3 -fold azimuthal anisotropy of the methyl groups in the SFG spectra was observed previously for octadecyl-terminated $\mathrm{Si}(111),{ }^{50}$ the situation when the alkyl chain was proposed to lie at $50^{\circ}$ and the terminal methyl group at $85^{\circ}$ with respect to the surface normal. ${ }^{50}$

Our experimental data could only be reproduced by radically changing the hyperpolarizability tensor $\beta^{(2)}$ of the $\mathrm{CH}_{3}$ symmetric stretch mode, introducing a $\beta_{a a a}^{(2)}$ component and significantly changing the ratio $\beta_{a a c}^{(2)} / \beta_{c c c}^{(2)}$ of the tensor elements, by a factor of $>20$ compared to the range of values $1.66-4.0$ reported in the literature for the methyl group. $22,37,38,43,47,51$ The observations that (1) azimuthal dependence of the $-\mathrm{CH}_{3}$ SFG signal cannot be simulated without significant alteration of the $-\mathrm{CH}_{3}$ hyperpolarizability tensor and (2) the resonant $-\mathrm{CH}_{3}$ signal shows the 3-fold azimuthal dependence in registry with the nonresonant signal of the silicon strongly suggest that the resonant molecular hyperpolarizability tensor $\beta^{(2)}$ of the surface-bound methyl is significantly affected by the $\operatorname{Si}(111)$ substrate. Indeed, it is reasonable to assume some degree of coupling between the $-\mathrm{CH}_{3}$ molecular vibrations and the electronic degrees of freedom in the underlying silicon. While the vibrational transition dipole moment $\left(\partial \mu_{c} / \partial q_{i}\right)$ in $\beta^{(2)}$, eq 16 , should not be strongly affected by the $\operatorname{Si}(111)$ surface, the polarizability $\alpha_{\mathrm{Si}}$ of the underlying electronic bath of the silicon substrate likely far exceeds the polarizability $\alpha_{\mathrm{Me}}$ of the methyl group itself because the polarizability scales with volume. Thus, even for a small coupling between the $\mathrm{C}-\mathrm{H}$ vibrations $q_{i}$ and the electronic polarizability $\alpha_{\mathrm{Si}}$ of the silicon, the polarizability derivative

$$
\frac{\partial \alpha}{\partial q_{i}}=\frac{\partial \alpha_{\mathrm{Si}}}{\partial q_{i}}+\frac{\partial \alpha_{\mathrm{Me}}}{\partial q_{i}}
$$

can be dominated by the bulk silicon response (the first term in eq 18).

On the basis of this consideration, the model for the nonresonant (electronic) response of $\mathrm{Si}(111)$ described above (Section 4.1) was adopted to describe the azimuthal dependence of the vibrationally resonant $\mathrm{CH}_{3}$ signal. An assumption made herein is that the vibrationally resonant SFG signal from the methyl stretch modes of the atop layer of $\mathrm{CH}_{3}$ groups has an isotropic component and has an anisotropic component of the same symmetry as the $\mathrm{Si}(111)$ surface. As in the case of the electronic response, both bulk and surface terms (eqs 7 and 9 and eqs 10 and 12, respectively) contribute to the resonant SFG signal. The bulk contribution to the vibrational signal is defined by two parameters, $\gamma_{i}$ and $\zeta_{i}$, which describe the 
coupling of the vibrational mode $i$ (ss or as) to the bulk electronic responses of silicon $\gamma$ and $\zeta$, eq 7. As shown in Figures 4,5 , and 8 , the experimental data can be modeled by assuming the ratio of $\gamma_{i} / \zeta_{i}$ to be the same as the ratio of $\gamma / \zeta$. To model the surface contribution (eq 10), the $\mathrm{CH}_{3}$ group is assumed to be oriented perpendicular to the $\mathrm{Si}(111)$ surface, implying that the $\mathrm{CH}_{3}$ transition dipoles for symmetric $\left(r^{+}\right)$and asymmetric $\left(r^{-}\right)$stretch modes are perpendicular and parallel to the surface, respectively. The $\mathrm{CH}_{3}$ hyperpolarizability tensor elements $\chi_{x x x}^{(2)}$ and $\chi_{z x x}^{(2)}$ must be zero for the $\mathrm{CH}_{3}$ symmetric stretch, $r^{+}$, and thus we assume that the surface contribution parameters $\partial_{11}^{\text {ss }}$ and $\partial_{31}^{\text {ss }}$ are zero, whereas $\partial_{15}^{\text {ss }}$ and $\partial_{33}^{\text {ss }}$ are assumed to be nonzero. Since $\partial_{11}^{\text {ss }}=0$ for the symmetric stretch, the anisotropy in the SFG $r^{+}$signal is therefore solely due to the Raman polarizability of the silicon bulk. Similarly, $\chi_{z z z}^{(2)}$ and $\chi_{x x z}^{(2)}$ are zero for the $\mathrm{CH}_{3}$ asymmetric stretch, $r^{-}$, that sets $\partial_{15}^{\text {as }}, \partial_{33}^{\text {as }}$ to zero and $\partial_{11}^{\text {as }}, \partial_{31}^{\text {as }}$ to nonzero elements

$$
\begin{aligned}
& \partial_{11}^{\text {ss }}=0, \partial_{31}^{\text {ss }}=0, \partial_{15}^{\text {ss }} \neq 0, \partial_{33}^{\text {ss }} \neq 0 \\
& \partial_{11}^{\text {as }} \neq 0, \partial_{31}^{\text {as }} \neq 0, \partial_{15}^{\text {as }}=0, \partial_{33}^{\text {as }}=0
\end{aligned}
$$

As shown by the solid blue lines in Figures 4, 5, and 8, the resonant SFG signals simulated using this model, eq 13, reproduce the modulation depth and phase of the azimuthal dependences of the symmetric and asymmetric $\mathrm{CH}_{3}$ stretch modes for both SSP and PPP polarization combinations (note that the phase of the azimuthal dependence is not adjustable). In analogy to nonresonant signal modeling, the ratio $R_{\mathrm{PPP}, \mathrm{SSP}}$ of the anisotropic and isotropic parts for the symmetric and asymmetric stretches can be evaluated (see Tables 4-6 of the Supporting Information). Two ratios for the $\mathrm{CH}_{3}$ symmetric stretch $R_{\mathrm{PPP}}^{\mathrm{ss}}=1.5$ and $R_{\mathrm{SSP}}^{\mathrm{ss}}=3.3$ are deduced. The $R_{\mathrm{PPP}}^{\mathrm{ss}}$ value was taken from the 300 fs delay PPP SFG resonant amplitude fit. For the $\mathrm{CH}_{3}$ asymmetric stretch (observed only in the PPP spectrum), one experimentally measured ratio, $R_{\mathrm{PPP}}^{\mathrm{as}}=1.5$, was determined. The $R_{\mathrm{PPP}, \mathrm{SSP}}$ ratios for the resonant vibrational response are in the same range as for the nonresonant electronic response that has been determined in this study and elsewhere, ${ }^{19}$ in accord with the assumption that the electronic polarizability of the $\mathrm{Si}$, modulated by the molecular vibrations of $\mathrm{CH}_{3}$, contributes to the response, per eq 18. As in the case of the nonresonant response, the fit does not provide a unique set of parameters but produces values for four parameters for every vibrational mode: two surface tensor elements and two bulk parameters $\gamma_{i}$ and $\zeta_{i}$. Table 6 in the Supporting Information presents one possible solution.

4.3. Rotational Dynamics. The azimuthal anisotropy of the $\mathrm{CH}_{3}$ resonant amplitude suggests that the methyl groups are not randomly oriented and also that they do not undergo free in-plane rotation. Instead, the 3-fold symmetry of the resonant signal suggests that the methyl hydrogens are pointing into three preferred directions, in registry with the crystal lattice of the $\mathrm{Si}(111)$ substrate. The data, however, do not preclude the possibility of hindered rotation proceeding via discrete $120^{\circ}$ jumps between the three preferred orientations.

The contribution of the orientational dynamics to the SFG spectroscopic line shapes is considered in detail elsewhere. ${ }^{52}$ Briefly, when orientational relaxation occurs on a time scale comparable to vibrational dephasing, the reorientation dynamics can be extracted from polarization-selected SFG line shape measurements. In particular, the in-plane rotation of the vertically oriented $\mathrm{CH}_{3}$ group should manifest itself as line broadening of the asymmetric stretch, whose transition dipole moment is parallel to the $\mathrm{Si}(111)$ surface, in the SPS spectrum relative to the PPP spectrum. ${ }^{52}$ Previously, the effect of reorientation dynamics on SFG amplitudes in the fast motion limit was considered by Wei and Shen ${ }^{53}$ and by Fourkas et al., ${ }^{54,55}$ who showed that fast rotational relaxation suppresses the SFG signal for the SPS polarization combination. The effective nonlinear susceptibility contributing to the SPS polarization SFG signal contains only one tensor element, $\chi_{y z y}^{(2)}$, which is selective to molecules with transition dipoles in the plane of the surface. Consistently, we observed the asymmetric $\mathrm{CH}_{3}$ stretch $\left(2976 \mathrm{~cm}^{-1}\right)$ in the SPS spectrum as well as in the PPP spectrum. Figure 9 superimposes the SPS and PPP spectra, showing that the $\mathrm{CH}_{3}$ asymmetric stretch line is broader in the SPS spectrum $\left(\Gamma_{\mathrm{SPS}}=15.9 \pm 1.0 \mathrm{~cm}^{-1}\right)$ than in the PPP spectrum $\left(\Gamma_{\mathrm{PPP}}=12.4 \pm 0.5 \mathrm{~cm}^{-1}\right)$.

Methyl-terminated $\mathrm{Si}(111)$ is a single-crystal surface that is likely to be nearly free of inhomogeneous broadening, corroborated by the spectral line shapes for both the PPP and SPS spectra being well fit by a Lorenztian profile, eq 4 . In this situation, the deconvolution of the instrument spectral resolution due to the time-delayed visible pulse is simplified, ${ }^{33}$ and Lorentzian half width at half-maximum $\Gamma$ can be approximated as a sum of the visible pulse width $\Gamma_{\text {vis }}$ the vibrational dephasing $\Gamma_{\text {vib }}$, and the rotational dephasing $\Gamma_{\text {rot }} 33,52$

$$
\Gamma=\Gamma_{\text {vis }}+\Gamma_{\text {vib }}+\Gamma_{\text {rot }}
$$

The narrow-band visible pulse, produced by an etalon in our measurement, decays exponentially in the time domain (see Supporting Information, Figure S2a) and thus is a Lorentzian in the frequency domain (see Supporting Information, Figure S2b) ${ }^{33}$ We also assume an exponential decay for the vibrational and rotational dephasing. The PPP spectrum has negligible contribution from the rotational relaxation and provides information mainly on the vibrational dephasing. ${ }^{52}$ Thus, when $\Gamma_{\text {vis }}=8.6 \mathrm{~cm}^{-1}$ is subtracted from $\Gamma_{\mathrm{PPP}}=12.4 \mathrm{~cm}^{-1}$, we obtained a value for the vibrational dephasing of $3.8 \mathrm{~cm}^{-1}$, or $\tau_{\text {vib }}=1 / \Gamma_{\text {vib }} \approx 1.4 \mathrm{ps}$, for the $\mathrm{CH}_{3}$ asymmetric stretch. The difference between the SPS and PPP line width provides the rotational dephasing of the methyl groups on the silicon surface as $\Gamma_{\text {rot }}=3.5 \pm 1.5 \mathrm{~cm}^{-1}$, which corresponds to a rotational relaxation time scale of $\tau_{\text {rot }} \sim 1-2$ ps.

It is interesting to compare the rotational dephasing of the methyl groups on $\mathrm{CH}_{3}-\mathrm{Si}(111)$ to the gas-phase rotational dephasing of a free methyl moiety. Assuming a quasi-classical Maxwellian distribution of free rotors, the rotational dephasing is described by the following correlation function $C_{1}(t)^{56}$

$$
\begin{aligned}
C_{1}(t) & =\left\langle P_{1}\left(\cos \theta_{t}\right)\right\rangle \\
& =\frac{I_{z}}{k_{\mathrm{B}} T} \int_{0}^{\infty} P_{1}[\cos (\Omega t)] \Omega \exp \left(-\frac{I_{z} \Omega^{2}}{2 k_{\mathrm{B}} T}\right) \mathrm{d} \Omega
\end{aligned}
$$

where $P_{1}$ is the first Legendre polynomial and $I_{z}$ is the moment of inertia for rotation about the molecular symmetry axis $\left(C_{3}\right.$ symmetry axis for methyl). From eq 21 the rotational dephasing for a free methyl rotor is calculated to be $\sim 100 \mathrm{fs}$ at room temperature. On $\mathrm{CH}_{3}-\mathrm{Si}(111)$ surfaces, the rotation of methyl groups on the $\mathrm{Si}(111)$ is thus slowed by an order of magnitude, suggesting a hindering potential for rotation. The rotational relaxation of methyl groups may proceed as $120^{\circ}$ jumps between the three equal minima of the rotational potential. Because the $\mathrm{CH}_{3}$ groups form a well-ordered, densely packed adlayer covalently bonded on $\mathrm{Si}(111)$, the rotation of 
$\mathrm{CH}_{3}$ groups about the $\mathrm{C}-\mathrm{Si}$ bonds might be interlocked, ${ }^{16}$ consistent with the observation of rotational anisotropy in the SFG signals reported herein. We note that while the resonant vibrational energy transfer between neighboring methyl groups is also a possible mechanism for orientational dephasing the weak transition dipole of the methyl group makes this mechanism unlikely. The Forster radius for methyl asymmetric stretch, i.e., the separation at which the rate of energy transfer through dipole-dipole coupling is comparable with the vibrational lifetime, is likely less than $1 \AA$ (for example, it is $2.1 \AA ̊$ in liquid water with its much higher $\mathrm{OH}$-stretch transition dipole $^{57}$ ), i.e., much smaller than the distance between methyls on the $\operatorname{Si}(111)$ surface, $\sim 3.8 \AA$.

\section{CONCLUSIONS}

Sum frequency generation has provided detailed information on the molecular structure and dynamics of $\mathrm{CH}_{3}$-terminated $\mathrm{Si}(111)$ surfaces. The vibrational $\mathrm{C}-\mathrm{H}$ stretch resonances in SFG spectra probe the orientation of the surface-bound methyl groups relative to the surface normal as well as relative to the crystalline axes of the silicon substrate, which is probed by the vibrationally nonresonant (electronic) response. The hyperpolarizability of the surface-bound methyl group was observed to be drastically different from that of free methyl groups, likely due to the coupling of the molecular vibration to the aboveband-gap Raman polarizability of the $\mathrm{Si}$ substrate. The propeller-like rotation of the methyl about the $\mathrm{Si}-\mathrm{C}$ bond was hindered, as evidenced by a pronounced 3-fold azimuthal anisotropy of the resonant SFG response. The data indicate that the methyl groups are primarily locked in one of three minima in registry with the 3 -fold symmetry of the $\mathrm{Si}(111)$ surface. Rotational motion occurred on a $1-2$ ps time scale, i.e., hindered by an order of magnitude compared to a free methyl rotor at room temperature ( $\sim 100 \mathrm{fs}$ rotational dephasing). The findings are consistent with a mechanism in which the methyl groups undergo $120^{\circ}$ jumps between the three equal rotational minima on the $\mathrm{CH}_{3}-\mathrm{Si}(111)$ surface.

\section{ASSOCIATED CONTENT}

\section{S Supporting Information}

Bulk and surface isotropic and anisotropic coefficients, fitting parameters, and figures of SFG geometry, visible pulse, and nonresonant suppression. This material is available free of charge via the Internet at http://pubs.acs.org.

\section{AUTHOR INFORMATION}

\section{Corresponding Author}

*E-mail: alex.benderskii@usc.edu. Phone: (213) 740-3220.

Notes

The authors declare no competing financial interest.

\section{ACKNOWLEDGMENTS}

This research was supported by the AFOSR, Grant No. FA9550-09-1-0547 (AVB), and by the NSF, CHE-0911682 (NSL). L.E.O. graciously thanks the financial support of the Link Foundation Energy Fellowship.

\section{REFERENCES}

(1) Shen, X. J.; Sun, B. Q.; Yan, F.; Zhao, J.; Zhang, F. T.; Wang, S. D.; Zhu, X. L.; Lee, S. High-Performance Photoelectrochemical Cells from Ionic Liquid Electrolyte in Methyl-Terminated Silicon Nanowire Arrays. ACS Nano 2010, 4, 5869-5876.
(2) Yates, J. T. A new opportunity in silicon-based microelectronics. Science 1998, 279, 335-336.

(3) Haick, H.; Hurley, P. T.; Hochbaum, A. I.; Yang, P. D.; Lewis, N. $\mathrm{S}$. Electrical characteristics and chemical stability of non-oxidized, methyl-terminated silicon nanowires. J. Am. Chem. Soc. 2006, 128, 8990-8991.

(4) Puniredd, S. R.; Assad, O.; Haick, H. Highly stable organic monolayers for reacting silicon with further functionalities: the effect of the $\mathrm{C}-\mathrm{C}$ bond nearest the silicon surface. J. Am. Chem. Soc. 2008, 130, 13727-34.

(5) Touahir, L.; Allongue, P.; Aureau, D.; Boukherroub, R.; Chazalviel, J. N.; Galopin, E.; Gouget-Laemmel, A. C.; de Villeneuve, C. H.; Moraillon, A.; Niedziolka-Jonsson, J.; Ozanam, F.; Andresa, J. S.; Sam, S.; Solomon, I.; Szunerits, S. Molecular monolayers on silicon as substrates for biosensors. Bioelectrochemistry 2010, 80, 17-25.

(6) Webb, L. J.; Lewis, N. S. Comparison of the electrical properties and chemical stability of crystalline silicon(111) surfaces alkylated using Grignard reagents or olefins with Lewis acid catalysts. J. Phys. Chem. B 2003, 107, 5404-5412.

(7) Royea, W. J.; Juang, A.; Lewis, N. S. Preparation of air-stable, low recombination velocity $\mathrm{Si}(111)$ surfaces through alkyl termination. Appl. Phys. Lett. 2000, 77, 1988-1990.

(8) Johansson, E.; Hurley, P. T.; Brunschwig, B. S.; Lewis, N. S. Infrared Vibrational Spectroscopy of Isotopically Labeled EthylTerminated Si(111) Surfaces Prepared Using a Two-Step Chlorination/Alkylation Procedure. J. Phys. Chem. C 2009, 113, 15239-15245.

(9) Webb, L. J.; Rivillon, S.; Michalak, D. J.; Chabal, Y. J.; Lewis, N. S. Transmission infrared spectroscopy of methyl- and ethyl-terminated silicon(111) surfaces. J. Phys. Chem. B 2006, 110, 7349-7356.

(10) Yu, H. B.; Webb, L. J.; Ries, R. S.; Solares, S. D.; Goddard, W. A.; Heath, J. R.; Lewis, N. S. Low-temperature STM images of methylterminated $\mathrm{Si}(111)$ surfaces. J. Phys. Chem. B 2005, 109, 671-674.

(11) Becker, J. S.; Brown, R. D.; Johansson, E.; Lewis, N. S.; Sibener, S. J. Helium atom diffraction measurements of the surface structure and vibrational dynamics of $\mathrm{CH} 3-\mathrm{Si}(111)$ and $\mathrm{CD} 3-\mathrm{Si}(111)$ surfaces. J. Chem. Phys. 2010, 133, 104705.

(12) Ferguson, G. A.; Raghavachari, K. The emergence of collective vibrations in cluster models: Quantum chemical study of the methylterminated Si(111) surface. J. Chem. Phys. 2006, 125, 154708.

(13) Solares, S. D.; Yu, H. B.; Webb, L. J.; Lewis, N. S.; Heath, J. R.; Goddard, W. A. Chlorination-methylation of the hydrogen-terminated silicon(111) surface can induce a stacking fault in the presence of etch pits. J. Am. Chem. Soc. 2006, 128, 3850-3851.

(14) Aliano, A.; Li, Y.; Cicero, G.; Galli, G. Structural and Electronic Properties of the Methyl-Terminated Si(111) Surface. J. Phys. Chem. C 2010, 114, 11898-11902.

(15) Yu, H. B.; Webb, L. J.; Heath, J. R.; Lewis, N. S. Scanning tunneling spectroscopy of methyl- and ethyl-terminated $\mathrm{Si}(111)$ surfaces. Appl. Phys. Lett. 2006, 88, 252111.

(16) Yamada, T.; Kawai, M.; Wawro, A.; Suto, S.; Kasuya, A. HREELS, STM, and STS study of CH3-terminated Si(111)- $(1 \times 1)$ surface. J. Chem. Phys. 2004, 121, 10660-10667.

(17) Tom, H. W. K.; Heinz, T. F.; Shen, Y. R. 2nd-Harmonic Reflection from Silicon Surfaces and Its Relation to Structural Symmetry. Phys. Rev. Lett. 1983, 51, 1983-1986.

(18) Sipe, J. E.; Moss, D. J.; Vandriel, H. M. Phenomenological Theory of Optical 2nd-Harmonic and 3rd-Harmonic Generation from Cubic Centrosymmetric Crystals. Phys. Rev. B 1987, 35, 1129-1141.

(19) Mitchell, S. A.; Boukherroub, R.; Anderson, S. Second harmonic generation at chemically modified $\mathrm{Si}(111)$ surfaces. J. Phys. Chem. B 2000, 104, 7668-7676.

(20) Hirose, C.; Yamamoto, H.; Akamatsu, N.; Domen, K Orientation Analysis by Simulation of Vibrational Sum-Frequency Generation Spectrum - Ch Stretching Bands of the Methyl-Group. J. Phys. Chem. 1993, 97, 10064-10069.

(21) Guyot-Sionnest, P.; Hunt, J. H.; Shen, Y. R. Sum-Frequency Vibrational Spectroscopy of a Langmuir Film: Study of Molecular 
Orientation of a Two-Dimensional System. Phys. Rev. Lett. 1987, 59, $1597-1600$.

(22) Zhuang, X.; Miranda, P. B.; Kim, D.; Shen, Y. R. Mapping molecular orientation and conformation at interfaces by surface nonlinear optics. Phys. Rev. B 1999, 59, 12632-12640.

(23) Richmond, G. L. Molecular bonding and interactions at aqueous surfaces as probed by vibrational sum frequency spectroscopy. Chem. Rev. 2002, 102, 2693-2724.

(24) Eisenthal, K. B. Liquid interfaces probed by second-harmonic and sum-frequency spectroscopy. Chem. Rev. 1996, 96, 1343-1360.

(25) Shen, Y. R.; Ostroverkhov, V. Sum-frequency vibrational spectroscopy on water interfaces: Polar orientation of water molecules at interfaces. Chem. Rev. 2006, 106, 1140-1154.

(26) Geiger, F. M. Second Harmonic Generation, Sum Frequency Generation, and chi $((3))$ : Dissecting Environmental Interfaces with a Nonlinear Optical Swiss Army Knife. Annu. Rev. Phys. Chem. 2009, 60, 61-83.

(27) Bain, C. D. Sum-Frequency Vibrational Spectroscopy of the Solid-Liquid Interface. J. Chem. Soc., Faraday Trans. 1995, 91, 12811296.

(28) Weeraman, C.; Yatawara, A. K.; Bordenyuk, A. N.; Benderskii, A. V. Effect of nanoscale geometry on molecular conformation: Vibrational sum-frequency generation of alkanethiols on gold nanoparticles. J. Am. Chem. Soc. 2006, 128, 14244-14245.

(29) Yatawara, A. K.; Tiruchinapally, G.; Bordenyuk, A. N.; Andreana, P. R.; Benderskii, A. V. Carbohydrate Surface Attachment Characterized by Sum Frequency Generation Spectroscopy. Langmuir 2009, 25, 1901-1904.

(30) Bansal, A.; Li, X. L.; Lauermann, I.; Lewis, N. S.; Yi, S. I.; Weinberg, W. H. Alkylation of $\mathrm{Si}$ surfaces using a two-step halogenation Grignard route. J. Am. Chem. Soc. 1996, 118, 7225-7226.

(31) Bansal, A.; Li, X. L.; Yi, S. I.; Weinberg, W. H.; Lewis, N. S. Spectroscopic studies of the modification of crystalline $\mathrm{Si}(111)$ surfaces with covalently-attached alkyl chains using a chlorination/ alkylation method. J. Phys. Chem. B 2001, 105, 10266-10277.

(32) O’Leary, L. E.; Johansson, E.; Brunschwig, B. S.; Lewis, N. S. Synthesis and Characterization of Mixed Methyl/Allyl Monolayers on Si(111). J. Phys. Chem. B 2010, 114, 14298-14302.

(33) Stiopkin, I. V.; Jayathilake, H. D.; Weeraman, C.; Benderskii, A. V. Temporal effects on spectroscopic line shapes, resolution, and sensitivity of the broad-band sum frequency generation. J. Chem. Phys. 2010, 132, 234503/1-234503/9.

(34) Lagutchev, A.; Hambir, S. A.; Dlott, D. D. Nonresonant background suppression in broadband vibrational sum-frequency generation spectroscopy. J. Phys. Chem. C 2007, 111, 13645-13647.

(35) Guyotsionnest, P.; Chen, W.; Shen, Y. R. General-Considerations on Optical 2nd-Harmonic Generation from Surfaces and Interfaces. Phys. Rev. B 1986, 33, 8254-8263.

(36) Bloember., N.; Chang, R. K.; Jha, S. S.; Lee, C. H. Optical Second-Harmonic Generation in Reflection from Media with Inversion Symmetry. Phys. Rev. 1968, 174, 813.

(37) Hirose, C.; Akamatsu, N.; Domen, K. Formulas for the Analysis of Surface Sum-Frequency Generation Spectrum by Ch Stretching Modes of Methyl and Methylene Groups. J. Chem. Phys. 1992, 96, 997-1004.

(38) Hirose, C.; Akamatsu, N.; Domen, K. Formulas for the Analysis of the Surface Sfg Spectrum and Transformation Coefficients of Cartesian Sfg Tensor Components. Appl. Spectrosc. 1992, 46, 10511072.

(39) Wang, H. F.; Gan, W.; Lu, R.; Rao, Y.; Wu, B. H. Quantitative spectral and orientational analysis in surface sum frequency generation vibrational spectroscopy (SFG-VS). Intl. Rev. Phys. Chem. 2005, 24, 191-256.

(40) Hong, S.-C.; Oh-e, M.; Zhuang, X.; Shen, Y. R.; Ge, J. J.; Harris, F. W.; Cheng, S. Z. D. Orientations of side chains and adsorbed liquid crystal molecules on a rubbed polyimide surface studied by optical second harmonic generation. Phys. Rev. E 2001, 63, 051706.

(41) Jayathilake, H. D.; Zhu, M. H.; Rosenblatt, C.; Bordenyuk, A. N.; Weeraman, C.; Benderskii, A. V. Rubbing-induced anisotropy of long alkyl side chains at polyimide surfaces. J. Chem. Phys. 2006, 125, 064706.

(42) Wei, X.; Zhuang, X.; Hong, S.-C.; Goto, T.; Shen, Y. R. SumFrequency Vibrational Spectroscopic Study of a Rubbed Polymer Surface. Phys. Rev. Lett. 1999, 82, 4256-4259.

(43) Hirose, C.; Yamamoto, H.; Akamatsu, N.; Domen, K. Orientation Analysis by Simulation of Vibrational Sum-Frequency Generation Spectrum - CH Stretching Bands of the Methyl-Group. J. Phys. Chem. 1993, 97, 10064-10069.

(44) Himmelhaus, M.; Eisert, F.; Buck, M.; Grunze, M. Self-assembly of n-alkanethiol monolayers. A study by IR-visible sum frequency spectroscopy (SFG). J. Phys. Chem. B 2000, 104, 576-584.

(45) Bordenyuk, A. N.; Jayathilake, H.; Benderskii, A. V. Coherent vibrational quantum beats as a probe of Langmuir-Blodgett monolayers. J. Phys. Chem. B 2005, 109, 15941-15949.

(46) Lu, R.; Gan, W.; Wu, B. H.; Zhang, Z.; Guo, Y.; Wang, H. F. C$\mathrm{H}$ stretching vibrations of methyl, methylene and methine groups at the vapor/alcohol $(n=1-8)$ interfaces. J. Phys. Chem. B 2005, 109, $14118-14129$.

(47) Wu, H.; Zhang, W. K.; Gan, W.; Cui, Z. F.; Wang, H. F. An empirical approach to the bond additivity model in quantitative interpretation of sum frequency generation vibrational spectra. $J$. Chem. Phys. 2006, 125, 133203.

(48) Ishiyama, T.; Sokolov, V. V.; Morita, A. Molecular dynamics simulation of liquid methanol. I. Molecular modeling including C-H vibration and fermi resonance. J. Chem. Phys. 2011, 134, 024509.

(49) Ishiyama, T.; Sokolov, V. V.; Morita, A. Molecular dynamics simulation of liquid methanol. II. Unified assignment of infrared, raman, and sum frequency generation vibrational spectra in methyl C$\mathrm{H}$ stretching region. J. Chem. Phys. 2011, 134, 024510.

(50) Nihonyanagi, S.; Miyamoto, D.; Idojiri, S.; Uosaki, K. Evidence for epitaxial arrangement and high conformational order of an organic monolayer on $\mathrm{Si}(111)$ by sum frequency generation spectroscopy. $J$. Am. Chem. Soc. 2004, 126, 7034-7040.

(51) Wolfrum, K.; Laubereau, A. Vibrational Sum-Frequency Spectroscopy of an Adsorbed Monolayer of Hexadecanol on Water Destructive Interference of Adjacent Lines. Chem. Phys. Lett. 1994, $228,83-88$.

(52) Vinaykin, M.; Benderskii, A. V. Contribution of Orientational Dynamics to Sum Frequency Spectroscopic Line Shapes. Submitted for publication, 2012.

(53) Wei, X.; Shen, Y. R. Motional effect in surface sum-frequency vibrational spectroscopy. Phys. Rev. Lett. 2001, 86, 4799-4802.

(54) Fourkas, J. T.; Walker, R. A.; Can, S. Z.; Gershgoren, E. Effects of reorientation in vibrational sum-frequency spectroscopy. J. Phys. Chem. C 2007, 111, 8902-8915.

(55) Rivera, C. A.; Fourkas, J. T. Reexamining the interpretation of vibrational sum-frequency generation spectra. Intl. Rev. Phys. Chem. 2011, 30, 409-443.

(56) McHale, J. L. Molecular spectroscopy, 1st ed.; Prentice Hall: Upper Saddle River, N.J., 1999.

(57) Woutersen, S.; Bakker, H. J. Resonant intermolecular transfer of vibrational energy in liquid water. Nature 1999, 402, 507-509. 